(C) 2006 IEEE. Reprinted, with permission, from X. Xiong, Y. Wu, and W. Jone, "Reliability Analysis of SelfRepairable MEMS Accelerometer", Proc. of 21th IEEE International Symposium on Defect and Fault Tolerance in VLSI Systems (DFT'06), pp. 236-244, Arlington, VA, USA, Oct. 2006.

This material is posted here with permission of the IEEE. Such permission of the IEEE does not in any way imply IEEE endorsement of any of the University of Bridgeport's products or services. Internal or personal use of this material is permitted. However, permission to reprint/republish this material for advertising or promotional purposes or for creating new collective works for resale or redistribution must be obtained from the IEEE by writing to pubs-permissions@ieee.org. By choosing to view this document, you agree to all provisions of the copyright laws protecting it. 


\title{
Reliability Analysis of Self-Repairable MEMS Accelerometer
}

\author{
Xingguo Xiong, Yu-Liang $\mathrm{Wu}^{*}$, and Wen-Ben Jone** \\ Department of Electrical and Computer Engineering, \\ University of Bridgeport, Bridgeport, CT 06604, USA \\ Department of Computer Science and Engineering*, \\ The Chinese University of Hong Kong, Shattin, Hong Kong \\ Department of ECECS, University of Cincinnati, Cincinnati, OH 45221, USA** \\ Email:xxiong@bridgeport.edu,ylw@cse.cuhk.edu.hk*,wjone@ececs.uc.edu**
}

\begin{abstract}
MEMS (Microelectromechanical System) yield and reliability have been a very critical issue. In our previous paper, we have proposed a self-repairable MEMS comb accelerometer device, and the yield analysis has demonstrated effective yield increase due to the BISR (built-in self-repair) design. In this paper, we developed a MEMS reliability model for quantitative assessment of the MEMS reliability analysis. Based on this model, we analyze the reliability of both non-BISR and BISR MEMS comb accelerometers under Z-axis shocking environment. Simulation results demonstrate very effective reliability enhancement due to the BISR design. The reliability model can also be applied to other MEMS devices under various failure mechanisms in a similar way.
\end{abstract}

Keywords: MEMS (Microelectromechanical System), redundancy repair, reliability, microaccelerometer, fracture probability.

\section{Introduction}

Yield and reliability are two major issues for the commercialization of MEMS technologies. With the commercialization of MEMS devices, their reliabilities must be thoroughly studied. Second, MEMS will be integrated into System-on-Chip (SoC) design very soon, so the reliability of an entire SoC cannot be guaranteed if the reliability of MEMS is low. Further, MEMS is finding more and more applications in safety-critical areas, such as aerospace, medical instruments, etc. For these applications, extremely high reliability is required. For example, during the launching process of a rocket, the failure of a tiny MEMS device may easily lead to unpredictable disasters. An unreliable bioMEMS chip embedded inside human body can be a serious threat to the health and may lead to the loss of life. Thus, reliability research for MEMS in safety-critical applications is an especially urgent need.

Efforts on MEMS failure mechanisms and reliability research have been reported [1]-[6]. In [1], a comprehensive analysis on various MEMS defect sources, failure mechanisms and how they affect the MEMS reliability have been proposed. In [2] [3], the MEMS material fatigue and aging under long-term cyclic loading has been discussed. In [4][5], MEMS reliability under shock and vibration environments has been explored. In [6], efforts have been made to explore the physical mechanism of stiction in surface micromachining and its impact on MEMS reliability. Although its importance has been widely recognized by researchers, MEMS reliability research still remains in its infant stage. Multiple energy domains are generally involved in the working principle of MEMS devices. Moreover, most MEMS devices contain movable components. Hence, MEMS devices are vulnerable to much more defect sources during its fabrication process and in-field usage compared to VLSI chips. This makes MEMS reliability research a challenging work. The understanding of various MEMS failure mechanisms is also non-trivial. Many MEMS failure mechanisms are unclear yet. The interaction among different MEMS failure mechanisms and its influence on MEMS reliability are not well discussed. Furthermore, most of the MEMS reliability analysis methods are still qualitative. A well-developed quantitative MEMS reliability model is not available.

In this work, we develop a MEMS reliability model which can be used to quantitatively evaluate the reliability of MEMS devices. The reliability model has been applied to quantitatively assess the reliability of our previously proposed self-repairable MEMS accelerometer [7]. Based on this model, we evaluate the reliabilities of both BISR (built-in self-repair) and non-BISR MEMS accelerometers under shock environments. 
A comparison of the simulation results demonstrates that the BISR design leads to an effective reliability enhancement when compared to the non-BISR device. Although here we analyze the MEMS reliability under shock environments as an example, the reliability model and the research strategies can also be applied to other MEMS devices under other failure mechanisms (e.g., material fatigue, stiction) in a similar way.

\section{Reliability Analysis of Non-BISR/BISR MEMS Accelerometers}

MEMS comb accelerometer device 1 contains a large number of finger groups which are necessary to ensure enough signal strength. However, the large number of finger groups unavoidably leads to the decrease in yield as well as reliability. In [7], we proposed a built-in self-repairable MEMS comb accelerometer device 3. The device consists of six identical modules (for example), and each module has its own beams, mass and finger structures (fixed and movable). By assumption, four modules are connected together as the main device, while the remaining two modules serve as redundancy. The movable parts of each module are physically connected to those of adjacent modules through the common anchors, and signals sensed by all movable fingers in the device are connected to the sensing circuit directly. However, the fixed fingers of each module are connected to the modulation signal circuit through switches made of analog MUXes. By turning on or off these switches, we can determine whether a module works as part of the main device or the redundant device. If a module is tested as faulty, the control circuit will permanently exclude the module from the main device and replace it with a good redundant module (if there is any). Thus, after repairing, the main device can still be ensured to work properly. Because each module has its own independent beam and mass structure, a faulty module does not affect the function of other modules. For example, even if the movable part of one module is broken or stuck to substrate, the movable parts of other modules can still move freely and work jointly to ensure the function of the main device. Our previous analysis in [7] demonstrates that the BISR design leads to effective yield increase. In this paper, we will develop a reliability model and evaluate the effectiveness of reliability enhancement due to the BISR design. In the following discussion, we call the MEMS comb accelerometer with (without) the BISR feature as a BISR (non-BISR) accelerometer. In order for a fair comparison, we assume that the total number of finger groups of the non-BISR accelerometer should equal to that of the main device in the BISR accelerometer.

\subsection{Basic Concepts of Reliability}

Reliability is the probability that a component, equipment, or system will perform the required function under different operating conditions encountered for a stated period of time [8]. The reliability function is denoted by $R(t)(0 \leq R(t) \leq 1)$ where $t$ is time. The larger the reliability function value is, the more reliable the component, equipment or system will be. Meanwhile, the unreliability $Q(t)$, or the probability of failure, is defined as the probability that a component, equipment, or system will not perform the required function under the operating conditions encountered for a stated period of time $t$. Apparently, we have $R(t)+Q(t)=$ 1. The failure rate $\lambda$ is expressed as the ratio of the total number of failures to the total operating time: $\lambda=\frac{K}{T}$, where $K$ is the number of failures and $T$ is the total operating time. The Mean Time To Failures (MTTF) is the reciprocal of the failure rate: $M T T F=\frac{1}{\lambda}$. In reality, the failure rate $\lambda$ is generally the function of time. The failure rate $\lambda(t)$ of mechanical components, VLSI chips and MEMS devices follows the behavior of a bathtub curve [8].

There are several standard probability models available for describing the reliability of a system [8]. Among them, the exponential reliability model is most frequently used. The reliability function $R(t)$ is expressed as

$$
R(t)=e^{-\lambda t}
$$

where $\lambda$ is the failure rate and $t$ is the time period. The unreliability $Q(t)$ is therefore

$$
Q(t)=1-R(t)=1-e^{-\lambda t} .
$$

The above reliability models describe the reliability of an individual component. However, sometimes we also need to evaluate the reliability of a system consisting of multiple components. Depending on the configuration of a system, different system reliability models (such as series, parallel, and k-out-of-n, etc.) are available [8]. The k-out-of-n redundancy reliability model is very useful for the reliability analysis of our BISR MEMS device. A k-out-of-n redundancy system consists of $n$ modules, among which at least $k$ modules need to be fault-free in order for the whole system to work properly. In other words, a maximum of $n-k$ 
faulty modules are allowed without losing the function of the whole system. Our BISR comb accelerometer [7] is exactly an example of k-out-of-n reliability model. If the BISR accelerometer contains $n$ modules in the main device and $m$ redundant modules, it is a n-out-of- $(\mathrm{n}+\mathrm{m})$ model. Assume the reliability of each component as $R_{c}$. The reliability $R_{t o t}$ of the k-out-of-n redundancy system is given as below [8]

$$
R_{\text {tot }}(t)=R_{c}^{n}(t)+n R_{c}^{n-1}(t)\left(Q_{c}\right)(t)+\ldots+\left(\begin{array}{c}
n \\
k
\end{array}\right)\left(R_{c}(t)\right)^{k}\left(Q_{c}(t)\right)^{n-k} .
$$

Assume the failure rate of each component as $\lambda$, the MTTF of the system can be calculated as [8]

$$
\operatorname{MTTF}=\frac{1}{\lambda}\left(\frac{1}{n}+\frac{1}{n-1}+\frac{1}{n-2}+\ldots+\frac{1}{k}\right) .
$$

\subsection{Reliability Model of Non-BISR MEMS Device}

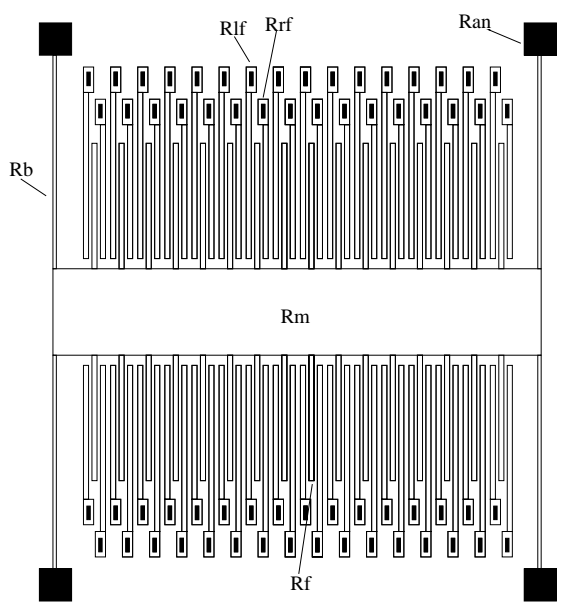

Figure 1. The structural diagram of a non-BISR MEMS device.

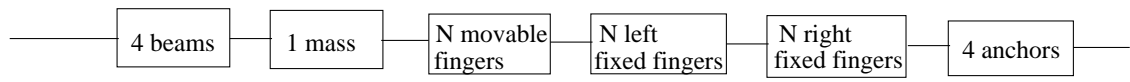

Figure 2. The reliability model for the non-BISR MEMS device.

The structural diagram of the non-BISR MEMS accelerometer [7][9] for this analysis is shown in Figure 1, and its corresponding reliability model is shown in Figure 2. As shown in Figure 1, the non-BISR device consists of four beams, one mass, $N$ number of movable fingers, $2 N$ number of left/right fixed fingers, and four anchors. All these components must be fault-free in order for the entire device to function correctly. If any of these components becomes faulty, the whole device will malfunction. For example, if one of the beams is broken, a movable finger is stuck to the corresponding fixed finger, or the mass is stuck to the substrate, then the device will not be able to work properly. Although the fixed components such as anchors and fixed fingers are less likely to be faulty than the movable components, they also must be fault-free in order to ensure the proper function of the device. For example, if one left fixed finger is shorted to its neighborhood right fixed finger, this will lead to a short circuit to the signal detection circuit. Or, if an anchor is lifted off the substrate, the device structure will be unstable. Thus, the reliability of a non-BISR device can be described as a series model, as shown in Figure 2. Since the four beams have exactly the same dimension and equal loading, we assume all four beams have the same reliability $R_{b}$. Similarly, we assume the reliabilities of the mass and each movable finger as $R_{m}$ and $R_{f}$ separately. The reliabilities of one fixed finger and one anchor are denoted as $R_{f f}$ and $R_{a n}$ individually. Finally, the reliability of the entire non-BISR device can be expressed as

$$
R_{n s r}=R_{b}^{4} \cdot R_{m} \cdot R_{f}^{N} \cdot R_{f f}^{2 N} \cdot R_{a n}
$$

In reality, movable components of the MEMS device are vulnerable to more defect sources when compared with fixed components. Thus, the reliabilities of fixed components can be much higher than those of movable components. The defects of fixed components mainly result from device fabrication. The devices with catastrophic defects in fixed components can be filtered out during manufacturing test. During 
in-field usage, it is less likely for the fixed components to develop new defects compared to the movable components. Thus, in the following discussion, we will mainly concentrate on the reliability of the movable components, and the reliability of the fixed parts can be treated as constant 1 . In this way, the reliability of the non-BISR device can be expressed as

$$
R_{n s r}=R_{b}^{4} \cdot R_{m} \cdot R_{f}^{N}
$$

From the reliability model of the non-BISR device, we can see that the major threat to the device reliability comes from the large index (i.e., $N$ ) of $R_{f}$. For example, if the reliability of a single movable finger is 0.99 and $N$ equals 42 , this will reduce the entire device reliability to 0.656 even though the beam and mass are assumed perfect. This is the major impetus for us to implement the redundancy repair technique for MEMS comb accelerometers. By modularizing the device, each module contains a smaller number of comb fingers. The reliability of each module will be higher than the original non-BISR device. By implementing the redundancy repair technique, even higher reliability can be achieved for the entire device.

Assume the failure rates for the beam, mass and finger as $\lambda_{b}, \lambda_{m}$ and $\lambda_{f}$ separately. The reliability functions of the beam, mass and finger can be expressed as

$$
R_{b}=e^{-\lambda_{b} t}, R_{m}=e^{-\lambda_{m} t}, R_{f}=e^{-\lambda_{f} t} .
$$

Hence, the reliability function of the non-BISR device with respect to time $t$ is

$$
R_{n s r}(t)=e^{-\lambda_{n s r} t}=e^{-\left(4 \lambda_{b}+\lambda_{m}+N \lambda_{f}\right) t} .
$$

where the failure rate $\lambda_{n s r}$ of the non-BISR device is $\lambda_{n s r}=4 \lambda_{b}+\lambda_{m}+N \lambda_{f}$.

Once we know the failure rates $\lambda_{b}, \lambda_{m}$ and $\lambda_{f}$, we can derive the reliability of the non-BISR device at certain time $t$ based on the equation for $R_{n s r}(t)$.

\subsection{Reliability model of BISR MEMS device}

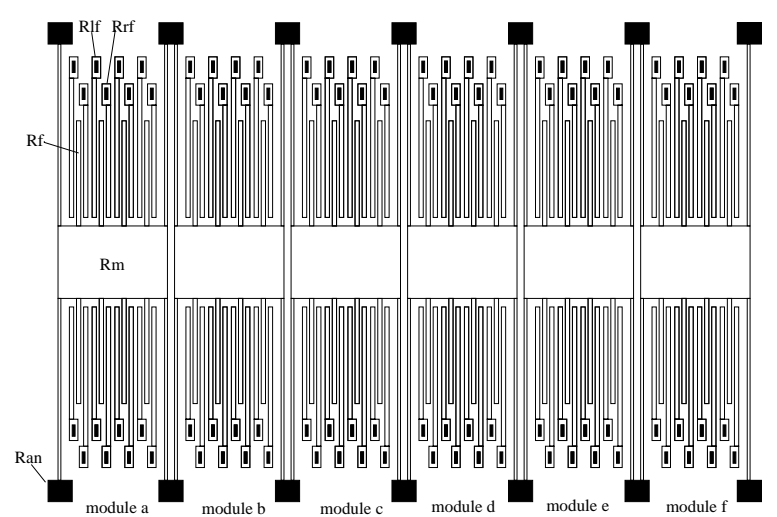

Figure 3. The structural diagram of the BISR MEMS accelerometer.

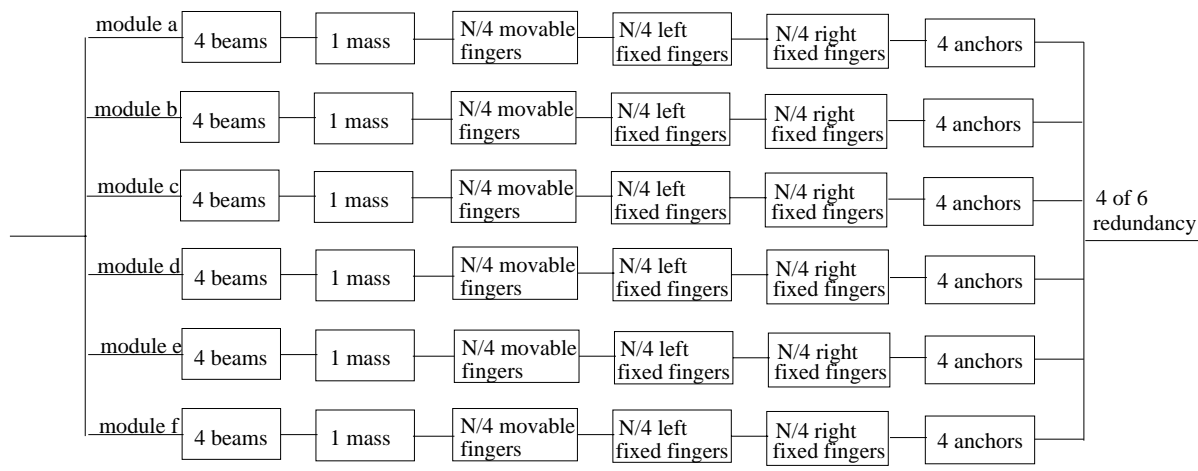

Figure 4. Reliability model for BISR device.

The structural diagram of the BISR comb accelerometer [7] is shown in Figure 3 and its corresponding reliability model is shown in Figure 4. The BISR comb accelerometer consists of six identical modules. In 
each module, it consists of four beams, one smaller mass, $(N / 4)$ number of movable fingers, $(N / 2)$ number of left and right fixed fingers, and four anchors. Note that $N$ is the number of movable fingers for the non-BISR device. Again, the reliability of each module can be described with a series model. Assume the reliabilities of each beam, movable finger and mass as $R_{b r}, R_{f r}$ and $R_{m r}$ separately, and the reliabilities of each fixed finger and anchor as $R_{f f}$ and $R_{a n}$ individually. The reliability $R_{m o d}$ of each BISR module can be expressed as

$$
R_{\text {mod }}=R_{b r}^{4} \cdot R_{m r} \cdot R_{f}^{N / 4} \cdot R_{f f}^{N / 2} \cdot R_{a n}
$$

Similarly, if we only concentrate on the reliability of movable components, the reliability of each module can be expressed as

$$
R_{m o d}=R_{b r}^{4} \cdot R_{m r} \cdot R_{f}^{N / 4}
$$

Assume the failure rates for the beam, mass and finger of each BISR module as $\lambda_{b r}, \lambda_{m r}$ and $\lambda_{f}$ separately. The reliability functions of the beam, mass and finger can be expressed as

$$
R_{b r}=e^{-\lambda_{b r} t}, R_{m r}=e^{-\lambda_{m r} t}, R_{f}=e^{-\lambda_{f} t} .
$$

Hence, the reliability function of the entire BISR module is

$$
R_{\text {mod }}(t)=e^{-\left(\lambda_{\text {mod }} t\right)}=e^{-\left[4 \lambda_{b r}+\lambda_{m r}+(N / 4) \lambda_{f}\right] t} .
$$

where the failure rate $\lambda_{\text {mod }}$ of each module is

$$
\lambda_{\text {mod }}=4 \lambda_{b r}+\lambda_{m r}+(N / 4) \lambda_{f} .
$$

The unreliability $Q_{\text {mod }}$ of each module is $Q_{\bmod }(t)=1-R_{\bmod }(t)$.

There are totally six identical modules in the BISR comb accelerometer. Among them, only four modules are required to be fault-free to ensure the proper function of the device. The reliability of such a system can be calculated using a k-out-of-n redundancy reliability model. The reliability $R_{s r}$ of the BISR accelerometer can be expressed as

$$
\begin{aligned}
R_{s r}(t) & =R_{\text {mod }}^{6}(t)+6 R_{\text {mod }}^{5}(t) Q_{\text {mod }}(t)+15 R_{\text {mod }}^{4}(t) Q_{\text {mod }}^{2}(t) \\
& =\left(e^{-\lambda_{\text {mod }} t}\right)^{6}+6\left(e^{-\lambda_{\text {mod }} t}\right)^{5}\left(1-e^{-\lambda_{\text {mod }} t}\right)+15\left(e^{-\lambda_{\text {mod }} t}\right)^{4}\left(1-e^{-\lambda_{\text {mod }} t}\right)^{2}
\end{aligned}
$$

This is the reliability function of a 4-out-of-6 redundancy BISR comb accelerometer. For the general case, assume there are $n$ number of modules in the main device and $m$ number of modules as redundancy. The reliability of the BISR accelerometer can be expressed as

$$
R_{s r}(t)=\left(R_{\text {mod }}(t)\right)^{n+m}+(n+m)\left(R_{\text {mod }}(t)\right)^{n+m-1}\left(Q_{\text {mod }}(t)\right)+\ldots+\left(\begin{array}{l}
n+m \\
n
\end{array}\right)\left(R_{\text {mod }}(t)\right)^{n}\left(Q_{\text {mod }}(t)\right)^{m} .
$$

\subsection{Reliability Enhancement and Reliability Analysis}

After we have developed the reliability models for both non-BISR and BISR MEMS devices, we can derive the reliability increase $I R(t)$ due to redundancy repair by

$$
I R(t)=R_{s r}(t)-R_{n s r}(t) .
$$

In the BISR MEMS comb accelerometer, the large number of comb finger groups are divided into several modules. Each module contains a smaller number of comb finger groups, and thus the risk for each module to be faulty is lowered. The redundancy further improves the reliability of the BISR device. However, compared to the original non-BISR device, the BISR device contains more beams. This will lead to the decrease of the reliability. The net reliability increase or decrease depends on the interaction between these counteracting factors. In fact, the reliability of a MEMS device is tightly linked to the corresponding defect sources and failure mechanisms. Different failure mechanisms lead to different device reliability behavior.

In order to evaluate the MEMS reliability, it is important to look into the possible MEMS failure mechanisms. In the following MEMS reliability analysis, we evaluate the reliabilities of example designs for both non-BISR and BISR devices under shock environment, and assume fracture as the major failure mechanism. The non-BISR device has a beam width of $W_{b n s r}=3.2 \mu \mathrm{m}$. For the BISR device, we consider two cases [10]: (1). BWC device: the BISR device with Beam Width Compensation (i.e., shrinking the beam width to compensate the sensitivity loss due to BISR design) with $W_{b b w c}=2.0 \mu m=0.63 W_{b n s r}$. (2). EFC device: the BISR device with Electrostatic Force Compensation (i.e., using electrostatic force to compensate the sensitivity loss) with $W_{b e f c}=3.2 \mu m=W_{b n s r}$. The design parameters of both the non-BISR and BISR devices are shown in Table 1. 
Table 1. Non-BISR/BISR accelerometers design parameters for reliability evaluation.

\begin{tabular}{|l|l|l|l|}
\hline \hline Design parameters & non-BISR device & BWC BISR module & EFC BISR module \\
\hline \hline beam width $W_{b}$ & $3.2 \mu \mathrm{m}$ & $2.0 \mu \mathrm{m}$ & $3.2 \mu \mathrm{m}$ \\
\hline beam length $L_{b}$ & $310 \mu \mathrm{m}$ & $310 \mu \mathrm{m}$ & $310 \mu \mathrm{m}$ \\
\hline mass width $W_{m}$ & $100 \mu \mathrm{m}$ & $100 \mu \mathrm{m}$ & $100 \mu \mathrm{m}$ \\
\hline mass length $L_{m}$ & $534 \mu \mathrm{m}$ & $132 \mu \mathrm{m}$ & $132 \mu \mathrm{m}$ \\
\hline movable finger width $W_{f}$ & $4 \mu \mathrm{m}$ & $4 \mu \mathrm{m}$ & $4 \mu \mathrm{m}$ \\
\hline movable finger length $L_{f}$ & $160 \mu \mathrm{m}$ & $160 \mu \mathrm{m}$ & $160 \mu \mathrm{m}$ \\
\hline number of finger groups $N$ & 48 & 12 & 12 \\
\hline device thickness $t$ & $2.0 \mu \mathrm{m}$ & $2.0 \mu \mathrm{m}$ & $2.0 \mu \mathrm{m}$ \\
\hline
\end{tabular}

\section{The Reliability of MEMS Accelerometer in Shock Survival}

In MEMS reliability research, it is very important to understand various failure modes and mechanisms for MEMS. Based on these, one can develop reliability models to quantitatively evaluate the MEMS reliability of each individual failure category, and find out the corresponding ways to improve the MEMS reliability. Some typical MEMS failure mechanisms [1] include material fatigue, mechanical fracture, stiction, wear, delamination, residual stress, etc. Further, environmentally induced failure mechanisms [4][5] are also important in MEMS failure. For example, shock, vibration, humidity, particle contamination, electrostatic discharge, etc. In this paper, we will focus our MEMS reliability under shock environment.

MEMS devices are designed to measure acceleration or other physical changes in a certain range. For example, MEMS accelerometers for automobile airbag deployment are designed to measure acceleration range between $\pm 50 \mathrm{~g}$. However, during the in-field usage, it cannot be guaranteed that the device will not experience acceleration beyond this range. Actually, in the lifetime of an accelerometer, it will always experience several times (or even more) of accidental shocks with extremely high acceleration [11]. In order to ensure high reliability, the device must be able to stand such accidental high-level shocks. Thus, it is very important to measure the device reliability under such high shocks. MEMS reliability under high shock environment has been researched recently [4][11][12]. In [11], the mechanical drop reliability test for ADXL50 accelerometers used a minimum of $4000 \mathrm{~g}$ shock from $1 \mathrm{~m}$ dropping onto concrete within $250 \mathrm{~ms}$. They also measured the device reliability in withstanding high accelerations in z-axis (direction perpendicular to the device plane) on a $30,000 \mathrm{~g}\left(294,210 \mathrm{~m} / \mathrm{s}^{2}\right)$ acceleration [11]. This value was chosen based on the maximum acceleration applied during centrifuge testing of the accelerometers. In [4], in order for the reliability test of a surface-micromachined micro-engine in shock environments, haversine shock pulses with widths of 1 to $0.2 \mathrm{~ms}$ in the range from $500 \mathrm{~g}$ to $40,000 \mathrm{~g}$ were used. Thus, in our experiment, we will also evaluate the shock reliability of both non-BISR and BISR devices in response to shock levels from $500 \mathrm{~g}$ to as high as $80,000 \mathrm{~g}$.

Shock can lead to the lift-off of a microstructure from the bonded surface. It can also lead to the adhesion and stiction of a movable microstructure to the substrate or fixed components. Intensive shock can also lead to the fracture of MEMS structures. The fracture behavior in turn can be described with Weibull fracture probability distribution [13]. Shock may lead to different results depending on its direction and strength. For example, a Z-axis shock toward $+Z$ direction may lead to the fracture of a beam, while a shock along $-Z$ direction may lead to the contact of movable components to the substrate. A shock toward X direction may lead to the contact and stiction of movable fingers to fixed fingers. However, a shock in Y direction can be less destructive except it is very strong. A MEMS reliability analysis in shock environments will be studied in detail for both non-BISR and BISR accelerometers in the following discussion.

\subsection{Assumptions}

In order to simplify the problem, we made several assumptions in the following discussion. First, we only consider the case of a negative Z-axis shock. That is, the shock acceleration is along the direction perpendicular to the device plane, and point to the negative $\mathrm{Z}$ direction. As a result, the movable microstructure will deflect along positive Z-axis, as shown in Figure 5. For a shock along the positive $\mathrm{Z}$ direction, it will lead to stiction between the movable structure and the substrate. Second, during high acceleration shock period, a packaged MEMS device may fail due to various defect sources. For example, the wire-bonding may be lifted off the anchor, or the debris/particles sealed in the package may fall out and move to capacitance gaps of the accelerometer, etc. These various defect sources may cause the device to be faulty in lower acceleration shock levels. However, for simplification, in this research we only concentrate on the structure fracture due to a large stress caused by the large displacement in high-g shocks. Since both ends of each beam 
are the most stress-concentrated area in MEMS comb acceleration, the structural fracture will first occur at the ends of each beam. Third, we ignore the air damping effect in the shock testing. But, we need to be aware that the air-damping effect may slightly alleviate the amplitude of the mass movement. Hence, it will help increase the durability of MEMS devices to high-g shocks. However, because the viscosity coefficient of air (gas) is very small, this effect is not significant, especially compared to the high-g shocks. Thus, air-damping effect will not be considered in our reliability comparison. Fourth, we assume both non-BISR and BISR devices are unpowered when the Z-axis shock is experienced. This means the devices will not have electrostatic force and the deflection of the movable microstructure is purely due to the applied acceleration shocks. Further, in traditional reliability models, the long-term reliability of a device is a function of time $t$. However, here we discuss the reliability of the device under an accidental shock event. Thus, we will use shock reliability $R_{f}$, which is the probability that a device can stand a given shock acceleration without fracture, to measure the device reliability. In this way, our device reliability $R_{f}$ is not a function of time $t$.

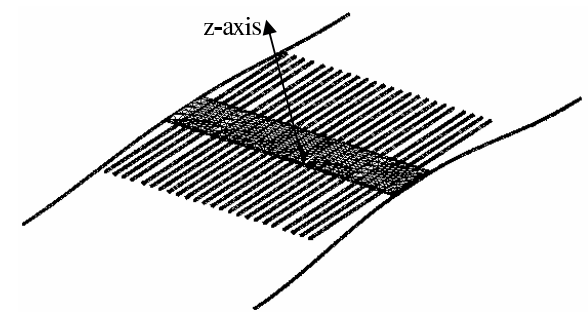

Figure 5. Deflection of accelerometer under Z-axis shock.

\subsection{Reliability Analysis for Shock}

In [13], the Weibull fracture probability function $P_{f}$ and the expected mean fracture stress $\sigma_{f}$ of a lateral accelerometer structure were derived. In a similar way, we can calculate the $P_{f}$ and $\sigma_{f}$ of our accelerometers. Since there is no frame tether in our accelerometer design, if we only consider the fracture behavior of one beam, there are two identical beam flank surfaces, i.e., the right and left side-walls of the beam. Thus, the Weibull fracture probability $P_{f}$ of one beam can be expressed as

$$
P_{f}=1-\exp \left[-\frac{W_{b} L_{b}}{m+1}\left(\frac{\sigma_{\max }}{\sigma_{0}}\right)^{m}\right] .
$$

where $W_{b}$ and $L_{b}$ are the width and the length of the beam, $m$ is the Weibull modulus, $\sigma_{\max }$ is the maximum stress in the beam when it is deflected under a given shock acceleration, and $\sigma_{0}$ is a normalizing factor. The Weibull modulus $m$ and the normalizing factor $\sigma_{0}$ are extracted by fitting the chi-squared method to the experimental measurement data [13]. For a given beam, its $W_{b}, L_{b}, m$ and $\sigma_{0}$ values are fixed. Thus, a larger maximum stress $\sigma_{\max }$ will lead to increased fracture probability $P_{f}$. When a MEMS accelerometer deflects under a given shock, the stress distribution is not uniform. For example, the beams experience a larger stress when compared to the mass due to larger deformation. Fracture is most likely to occur first in the region with the maximum stress level. Thus, the device fracture probability will be decided by the maximum stress in the device structure, which is somewhere in the beams. The maximum stress $\sigma_{\max }$ in the beam under a given acceleration shock can be extracted by ANSYS simulation.

For reliability analysis, first we extract the maximum stress in the non-BISR device and one BISR module respectively using ANSYS, in response to a given acceleration. Then, we use the Weibull distribution function to derive the fracture probability $P_{f b n s r}$ of one beam for the non-BISR device, and the fracture probability $P_{f b m o d}$ of one beam in a BISR module. The shock reliability, $R_{f n s r}$, of the non-BISR device under a given shock acceleration, can be calculated as:

$$
R_{f n s r}=\left(1-P_{\text {fbnsr }}\right)^{4} .
$$

The shock reliability $R_{f m o d}$ of one BISR module can be derived by:

$$
R_{f m o d}=\left(1-P_{f b m o d}\right)^{4} .
$$

The reliability of the entire BISR device can be calculated based on the 4-out-of-6 redundancy reliability model derived before by:

$$
R_{s r}=R_{f \bmod }^{6}+6 R_{f \bmod }^{5}\left(1-R_{f m o d}\right)+15 R_{f \bmod }^{4}\left(1-R_{f m o d}\right)^{2} .
$$


The z-shock reliability increase IR of the comb accelerometer can be calculated by

$$
I R=R_{s r}-R_{n s r} .
$$

The above discussion gives our strategy to evaluate the reliabilities of both non-BISR and BISR devices for a shock failure mechanism. Assume an equal shock acceleration is applied to both BISR and non-BISR devices. Compared to the non-BISR device, the EFC-BISR module gives smaller amount of displacement in the unpowered condition due to the reduced sensing mass, which leads to the reduced maximum stress $\sigma_{\max }$. For each BWC-BISR module, the shock acceleration gives the same amount of displacement as the non-BISR device due to beam width compensation. However, because the beam width is shrunk, the maximum stress $\sigma_{\max }$ is also reduced due to beam width shrinking. Thus, for both EFC and BWC BISR modules, the fracture probability of one beam will be reduced. That is, $P_{f b m o d} \leq P_{f b n s r}$. The redundancy repair will lead to further reliability enhancement, and we can expect reliabilty $R_{s r}$ to be larger than $R_{n s r}$.

\subsection{Reliability Analysis Result}

We used ANSYS simulation to extract the maximum stress $\sigma_{\max }$ values corresponding to about 30 different Z-axis shock accelerations from $0 g$ to $10^{5} \mathrm{~g}$. Based on these data, we can calculate the Weibull fracture probability $\left(P_{f}\right)$ and the shock reliability $\left(R_{f}\right)$ values for non-BISR and BWC/EFC BISR devices corresponding to each individual shock acceleration. Relationships for the Weibull fracture probability of one beam versus each Z-axis shock acceleration for non-BISR/BISR devices are shown in Figure 6. The plots of the simulated Z-axis shock reliability versus each shock acceleration of non-BISR/BISR devices are shown in Figure 7. From Figure 6, it is clearly shown that the fracture of the beams of the BISR modules occurs at much higher acceleration, compared to the non-BISR device. That is, the maximum Z-axis shock acceleration the accelerometer can withstand is greatly extended due to the redundancy repair design.

As shown in the Figure 7, the shock reliability of the EFC BISR device is larger than that of the BWC BISR device, and both are larger than the non-BISR device. This result is intuitive because, in each BISR module of both BWC and EFC devices, the mass is reduced to 1/4 that of the non-BISR device. In the BWC BISR device, the beam thickness remains the same, but the beam width is shrunk to 0.63 that of the non-BISR device. That is, the Z-direction spring constant reduces to 0.63 that of the non-BISR value. This translates to a smaller Z-direction displacement and smaller maximum stress in the BISR device under the same Z-axis acceleration input. For the EFC BISR device, the beam spring constant remains the same as that of the nonBISR device. Thus, the maximum stress for the EFC BISR device is even smaller than that of the BWC BISR device. Hence, the maximum Z-axis acceleration shock the BISR devices can withstand is greatly increased. We can see that if the Z-shock acceleration falls into the range between the mean fracture stress of non-BISR device and BISR devices, a (maximum) reliability increase as large as 1 can be achieved due to the BISR design.

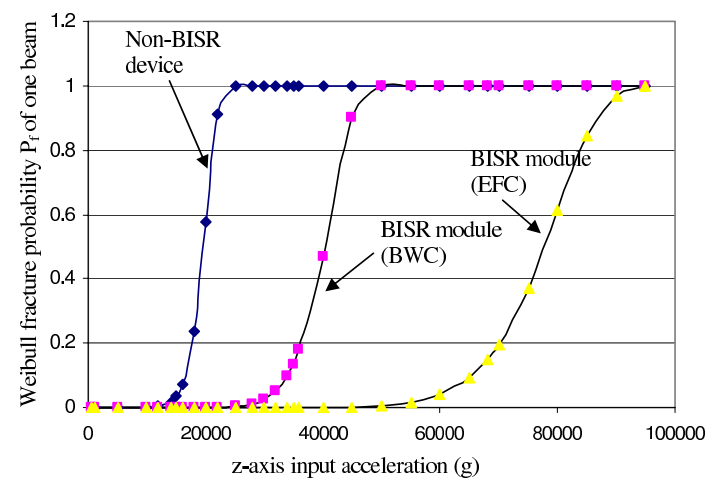

Figure 6. The Weibull fracture probabilities for non-BISR device and BISR modules.

\section{Conclusion and Future Research}

In this paper, a quantitative MEMS reliability model has been developed. Based on this reliability model, the reliabilities of both non-BISR and BISR MEMS comb accelerometer devices under shock environment have been assessed. The Weibull fracture probability function has been used to evaluate the fracture probability of MEMS accelerometers under Z-axis shock acceleration. The simulation results demonstrate an effective 


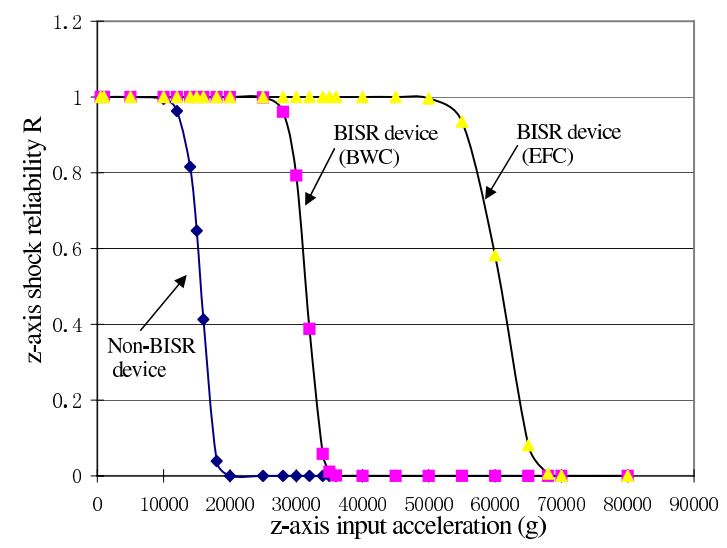

Figure 7. The z-axis shock reliability for non-BISR/BISR devices.

reliability enhancement for both the BWC and EFC BISR comb accelerometers, compared to non-BISR design. This verifies the effectiveness of the BISR design in MEMS reliability improvement. The MEMS reliability analysis under other failure mechanisms (such as material fatigue, stiction.) has also been explored. The corresponding results will be published separately. In the future research, we will consider the effect of process variation on MEMS reliability, such as the effect of beam width variation, material mechanical property variation, etc. We will also try to further improve our MEMS reliability model to account for other MEMS devices under various failure mechanisms. In this way, the reliability model can be used to assess and predict the MEMS reliability for various MEMS devices under various failure mechanisms. It can also guide us in improving the MEMS reliability for safety-critical applications.

\section{References}

[1] B. Stark (editor), "MEMS Reliability Assurance Guidelines for Space Applications”, Jet Propulsion Laboratory Publication 99-1, Pasadena, USA, Jan. 1999.

[2] M. Tabib-Azar, K. Wong, and W. Ko, "Aging Phenomena in heavily doped ( $\mathrm{p}+$ ) micromachined silicon cantilever beams", Sensors and Actuators A, Vol. 33, pp. 199-206, 1992.

[3] C. L. Muhlstein, R. T. Howe, and R. O. Ritchie, "Fatigue of polycrystalline silicon for microelectromechanical system applications: crack growth and stability under resonant loading conditions", Mechanics of Materials, Vol. 36, pp. 13-33, 2004.

[4] D. M. Tanner, J. A. Walraven, K. Helgesen, L. W. Irwin, F. Brown, N. F. Smith, and N. Masers, ”MEMS reliability in shock environments", Proceedings of IEEE International Reliability Physics Symposium, San Jose, CA, USA, pp. 129-138, Apr. 10-13, 2000.

[5] D. M. Tanner, J. A. Walraven, K.S.Helgesen, L. W. Irwin, D. L. Gregory, J. R. Stake, and N. F. Smith, "MEMS reliability in a vibration environment", IEEE 38th Annual International Reliability Physics Symposium, San Jose, California, USA, pp. 139-145, 2000.

[6] W. M. V. Spengen, R. Puers, and I. D. Wolf, "On the physics of stiction and its impact on the reliability of microstructures", Journal of Adhesion Science and Technology, Vol. 17, No. 4, pp. 563-582, 2003.

[7] X. Xiong, Y. Wu, and W. Jone, "Design and analysis of self-repairable MEMS accelerometer," Proceedings of the 20th IEEE International Symposium on Defect and Fault Tolerance in VLSI Systems (DFT'05), Monterey, CA, USA, pp. 21-29, Oct. 3-5, 2005.

[8] K. Neubeck, Practical Reliability Analysis, Pearson Prentice Hall, New Jersey, USA, 2004.

[9] W. Kuehnel, and S. Sherman, "A Surface Micromachined Silicon Accelerometer with On-chip Detection Circuitry," Sensors and Actuators, Vol. A, Issue 45, pp. 7-16, 1994.

[10] X. Xiong, Built-in self-test and self-repair for capacitive MEMS devices, Ph.D dissertation, University of Cincinnati, July 2005.

[11] S. Bart, J. Chang, T. Core, L. Foster, A. Olney, S. Sherman, and W. Tsang, "Design rules for a reliable surface micromachined IC sensor", Proceedings of 33rd Annual IEEE International Reliability Physics, pp. 311-317, Apr. 1995.

[12] T. G. Brown and B. Davis, "Dynamic high-g loading of MEMS sensor: ground and flight testing", SPIE Proceedings, Vol. 3512, pp. 228-235, 1998.

[13] S. Greek, F. Ericson, S. Johansson, and J. Schweitz, "In situ tensile strength measurement and Weibull analysis of thick film and thin film micromachined polysilicon structure", Thin Solid Films, Vol. 292, pp. 247-254, 1997. 